\title{
Análise retrospectiva da incidência de neoplasias em Vassouras/RJ
}

\author{
Retrospective analysis of cancer incidence in Vassouras/RJ
}

\author{
Alex Pereira Ramos*, Sebastião Jorge da Cunha Gonçalves
}

Como citar esse artigo. Ramos,

AP; Gonçalves, SJC. Análise retrospectiva da incidência de neoplasias em Vassouras/RJ. Revista de Saúde. 2018 Jul./Dez.; 09 (2): 02-08

\begin{abstract}
Resumo
A As elevadas taxas de prevalência e incidência de neoplasias têm sido alvos de debates que têm objetivo o planejamento de estratégias de saúde que visem a diminuição desses índices. Através do SISPACTO, estabeleceu-se que deveria haver a diminuição de $2 \%$ do indicador de saúde (indicador 30a) quando fosse comparado com o ano anterior. Em Vassouras, entre 2007 até 2016, essa meta só foi alcançada três vezes, sendo que em apenas uma oportunidade houve queda da incidência. O objetivo desse trabalho foi ana-lisar retrospectivamente o número de casos de neoplasias no município de Vassouras/RJ, evidenciando políticas de saúde do município para controle dessas doenças, comparando também à incidência estadual. A incidência de neoplasias malignas em Vassouras no período de 2007 a 2016 foi de 492 casos por 100.000 habitantes, um valor considerado alto, ainda mais quando em comparação com a incidência das doenças em todo o estado do Rio de Janeiro (214 por 100.000, no período de 2008 a 2016). Em Vassouras/RJ, a política de controle vem funcionado de modo interdisciplinar, respeitando a realidade da saúde nacional no aspecto condizente à importância do foco na saúde da mulher e do homem. Não há preconização, além das estabelecidas pelo Ministério da Saúde e INCA, para as neoplasias visualizadas no sexo masculino, categoria historicamente mais difícil de se abordar nas estratégias de saúde. Percebeu-se através dessa análise que a evolução do câncer em Vassouras se deu de forma ímpar, necessitando de adaptações para sua epidemiologia para que haja um melhor controle nos fatores causais.
\end{abstract}

Palavras-chave: Epidemiologia; Câncer; Políticas de Saúde.

\begin{abstract}
The high rates of prevalence and incidence have been the target of many debates, having focus on planning health strategies aimed at reducing these indicators. By the SIS-PACTO, it was established the health indicator (indicator 30a) must have a decrease of 2\% when compared to its previous year. In Vassouras, between 2007 to 2016, this goal was reached only three times; indeed, having fallen once. Thus, the objective of this study was to analyse retrospectively the number of cases of cancer in Vassouras/RJ, showing the municipal health policies to control these diseases, also comparing to the incidence in Rio de Janeiro state. The incidence of cancer in Vassouras between 2007 to 2016 was 492 cases/100.000 inhabitants, a high-level number, especially when compared to the incidence of cancer in the state of Rio de Janeiro (214/100.000 between 2008 to 2016). In Vassouras/RJ, the disease control is operated in an interdisciplinary way, respecting the reality of the national health, consonant to the importance of the focus on the women and men health. There is no recommendation, other than those established by the Ministério da Saúde and INCA, for common male's types of cancer, a historically more difficult category in health strategies. It can be seen from this analysis that the evolution of cancer in Vassouras occurred in a unique way, necessitating adaptations on its epidemiology so that there is a better control in the causal factors.
\end{abstract}

Keywords: Epidemiology; Cancer; Health Politics.

\section{Introdução}

A epidemiologia mundial das principais causas de morbidade e mortalidade permite atribuir ao câncer uma posição de importância nas políticas de saúde pública. Com a evolução do conhecimento científico a respeito do surgimento de neoplasias, as elevadas taxas de prevalência e incidência têm sido alvos de debate, tendo como objetivo o planejamento de estratégias de saúde que visem a diminuição desses índices.
Sabe-se que atualmente a principal causa de aumento do número de casos de neoplasias se dá através do aumento da expectativa de vida e, por conseguinte, o da idade populacional ${ }^{1}$. Tal fator, agregado ao estilo de vida da sociedade moderna (tabagismo, etilismo, sedentarismo, obesidade, dentre outros), constitui um importante fator causal para o aumento das neoplasias, devendo, portanto, estarem presente nas ações de combate ao câncer ${ }^{2}$.

Globalmente, há uma diferente distribuição dos 
tipos de neoplasias. Enquanto nos países desenvolvidos as neoplasias de pulmão e intestino são os tipos de maior incidência atualmente ${ }^{3}$, no Brasil as neoplasias de mama e próstata são as mais comuns (excluindose as neoplasias benignas de pele $)^{4}$. Esta diferença na epidemiologia demográfica não é exclusiva entre países desenvolvidos ou não: diferentes regiões de um mesmo país apresentam incidências diversas de neoplasias, evidenciando possíveis falhas de políticas de saúde e/ ou maior exposição a um fator causal. Assim, o estudo individual das neoplasias de cada região é essencial ao controle mais rigoroso do aumento da incidência.

No Brasil, as políticas nacionais de combate ao câncer visam muito além da prevenção secundária da doença; prevenção essa que já foi bastante popularizada no mundo moderno, levando ao aumento exponencial de diagnóstico de neoplasias, como foi o caso da relação de PSA sérico e câncer de próstata ${ }^{5}$. No país, a promoção em saúde tem um foco especial na prevenção primária, tentando evitar que a doença sequer chegue a acontecer.

Com essa preocupação, foi determinado a confecção do SISPACTO, um sistema que permite o registro de metas individualizadas e pactuadas para os municípios. No que diz respeito à categoria de doenças crônicas não transmissíveis, que inclui as neoplasias, estabeleceu-se que deveria haver a diminuição de $2 \%$ do indicador de saúde (indicador 30a) quando fosse comparado com o ano anterior.

No município de Vassouras não houve diminuição desse indicador, acontecendo, inclusive, aumento de 23\% (comparação de 2016 com 2015). Entre 2008 até 2016, essa meta só foi alcançada três vezes, sendo que em apenas uma oportunidade houve queda da incidência - entre os anos de 2010 e 2011, houve redução do índice em 3,15\% ${ }^{6}$. Sendo assim, faz-se necessário uma análise mais criteriosa dos tipos mais prevalentes de câncer no município e políticas atuais de saúde do município para redução desse indicador. O objetivo desse trabalho foi analisar retrospectivamente o número de casos de neoplasias no município de Vassouras/RJ, evidenciando políticas de saúde do município comparado à incidência estadual.

\section{Material e métodos}

Este estudo foi realizado a partir de uma pesquisa retrospectiva longitudinal quantitativa sobre os números de casos de neoplasias do município de Vassouras. A busca foi através do site DATASUS, Epidemiologia TABNET, sobre morbidade no Estado do Rio de Janeiro. Buscou-se o número bruto de neoplasias (CID C00 a C97) no município de Vassouras/RJ, nos anos entre 2008 e 2017 (sendo este último tendo contabilização apenas até o mês de abril), estipulando-se limites preconizados, como o de idade (30-69 anos), para cálculo do indicador.

Os valores foram utilizados a partir de tabelamento fornecido pelo sistema do DATASUS, através do Microsoft Office Excel 2013; este mesmo programa foi usado para cálculo de porcentagem simples do indicador de saúde do município, respeitando a fórmula matemática preconizada pelo SISPACTO. Para os valores esperados dos indicadores de saúde foram utilizadas as referências no Caderno de Diretrizes Objetivos, Metas e Indicadores 2017-2021 (Indicador 30a) e a Pactuação Individual de Diretrizes, Objetivos, Metas e Indicadores - 2017, do município.

A revisão de literatura acerca do tema foi feita através dos principais sites de indexação de arquivos (Google Acadêmico, Scielo, Lilacs e Pubmed), publicados a partir de 2008, data compatível com o ano de início das análises dos casos. A adição de artigos anteriores a essa data foi utilizada devido à necessidade de o estudo abranger políticas de saúde de prevenção do câncer. Os descritores de busca foram "câncer", "câncer no Brasil", "epidemiologia do câncer", "políticas de saúde no câncer", "prevenção do câncer", dentre outros. Os termos listados anteriormente são em português, mas os mesmos foram traduzidos na língua original de busca dos artigos para que pudesse achar artigos que fossem de valor à pesquisa, principalmente aqueles que falavam sobre políticas inovadoras de prevenção ao câncer em vários locais do mundo, até mesmo em outros municípios brasileiros.

\section{Resultados}

Através dos dados disponibilizados pela plataforma do DATASUS, pode-se obter o número de casos total de neoplasias no ano de 2016, no município de Vassouras/RJ e no estado do Rio de Janeiro (Tabela 1). Conforme tabela 1, observa-se o ranking das cinco neoplasias mais frequentes nas localidades, podendo ser feita uma comparação das duas situações. Foram excluídas, entretanto, algumas categorias que não se encontraram de acordo com os critérios de inclusão disponibilizados pelo SISPACTO (casos de neoplasias entre 30 e 69 anos, com CID 10: C00-C97).

Dado isso, exclui-se o número de casos de algumas categorias, como leiomioma uterino, que, embora se encontre na classificação de neoplasias do sistema, não se inclui no CID 10 de neoplasia maligna. Outras categorias também foram excluídas, entretanto sua ausência nas estatísticas não interfere diretamente na relevância dos dados.

As neoplasias malignas mais comuns nos homens e nas mulheres em Vassouras no ano de 2016 foram câncer de cólon e câncer de mama respectivamente (Tabela 1). Uma diferença em comparação com o 
Tabela 1. Número de Casos dos Cinco Tipos de Neoplasias Malignas mais Comuns em Vassouras/RJ e no estado do Rio de Janeiro, em 2016.

\begin{tabular}{|c|c|c|c|c|}
\hline & $\begin{array}{l}\text { Homens/Vassouras } \\
\text { (CID } / n^{\circ} \text { de casos) }\end{array}$ & $\begin{array}{l}\text { Mulheres/Vassouras } \\
\text { (CID } / n^{\circ} \text { de casos) }\end{array}$ & $\begin{array}{c}\text { Homens/Rio de } \\
\text { Janeiro } \\
\text { (CID } / \mathrm{n}^{\circ} \text { de casos) }\end{array}$ & $\begin{array}{l}\text { Mulheres/Rio de } \\
\text { Janeiro } \\
\text { (CID } / \mathrm{n}^{\circ} \text { de casos) }\end{array}$ \\
\hline $1^{\circ}$ & C18 (21) & $\overline{C 50(81)}$ & $\begin{array}{c}\text { C61 } \\
(2.346)\end{array}$ & $\begin{array}{c}\text { C50 } \\
(6.267)\end{array}$ \\
\hline $2^{\circ}$ & C67 (15) & Cl8 (14) & $\begin{array}{c}\text { C18 } \\
(1.225)\end{array}$ & $\begin{array}{c}\text { C53 } \\
(1.643)\end{array}$ \\
\hline $3^{\circ}$ & C19 (15) & C19 (13) & $\begin{array}{l}\text { C91-3 } \\
(1.200)\end{array}$ & $\begin{array}{c}\text { C57 } \\
(1.183)\end{array}$ \\
\hline $4^{\circ}$ & Cl6 (14) & $\mathrm{Cl6}(08)$ & $\begin{array}{l}\text { C19 } \\
(933)\end{array}$ & $\begin{array}{l}\text { C19 } \\
(969)\end{array}$ \\
\hline $5^{\circ}$ & C6l (12) & C57 (08) & $\begin{array}{l}\text { C34 } \\
(917)\end{array}$ & $\begin{array}{l}\text { C91-3 } \\
(900)\end{array}$ \\
\hline
\end{tabular}

CD16: Neoplasia Maligna de Estômago CD18: Neoplasia Maligna de Cólon CD19: Neoplasia Maligna da Junção Sigmoide C34: Neoplasia Maligna dos Brônquios e dos Pulmões CD50: Neoplasia Maligna de Mama C53: Neoplasia Maligna do Colo de Útero C57: Neoplasia Maligna de Outros Órgãos Genitais Femininos C61: Neoplasia Maligna de Próstata C67: Neoplasia Maligna de Bexiga C91-3: Leucemia Fonte: Datasus6

perfil epidemiológico dos homens do Estado do Rio de Janeiro, onde a neoplasia mais comum foi o câncer de próstata; está em $5^{\circ}$ lugar nos homens de Vassouras.

Em um aspecto geral, a incidência de neoplasias malignas em Vassouras no período de 2007 a 2016 foi de 492 casos por 100.000 habitantes, um valor considerado alto, ainda mais quando em comparação com a incidência das doenças em todo o estado do Rio de Janeiro (214 por 100.000 , no período de 2008 a 2016). Os valores totais de casos podem ser vistos no Figura 1.

Apesar de Vassouras apresentar uma maior incidência de neoplasias malignas nos dez anos observados quando comparada ao Estado do Rio de Janeiro, não há indícios de que haja um novo fator atuante durante o período que mantenha o crescimento da cidade maior que o crescimento do estado. Isso possibilita duas hipóteses situacionais muito importantes acerca da epidemiologia do câncer em Vassouras. A primeira delas é que a alta incidência é um agente crônico, estando presente há pelo menos dez anos no município. A segunda, e a mais alarmante como proposta, é que provavelmente as medidas de prevenção à saúde no município não estão fazendo efeito da forma esperada - causando redução da incidência - muito embora, como descrito anteriormente, os valores entre as duas regiões não apresentaram significância estatística. A razão dessas hipóteses será discutida no momento da discussão.

No que diz respeito ao indicador 30a, o município de Vassouras não alcançou as metas preconizadas em alguns anos, como pode ser visto na Tabela 2. Isso serve como ratificação à hipótese de que as políticas de prevenção em saúde do município não estão atingindo os objetivos da forma que deveriam, justificando, portanto, a adoção de novos métodos de promoção em saúde, principalmente aqueles que visem a redução dos principais tipos de neoplasias do município, deixando as políticas em saúde mais direcionadas aos problemas

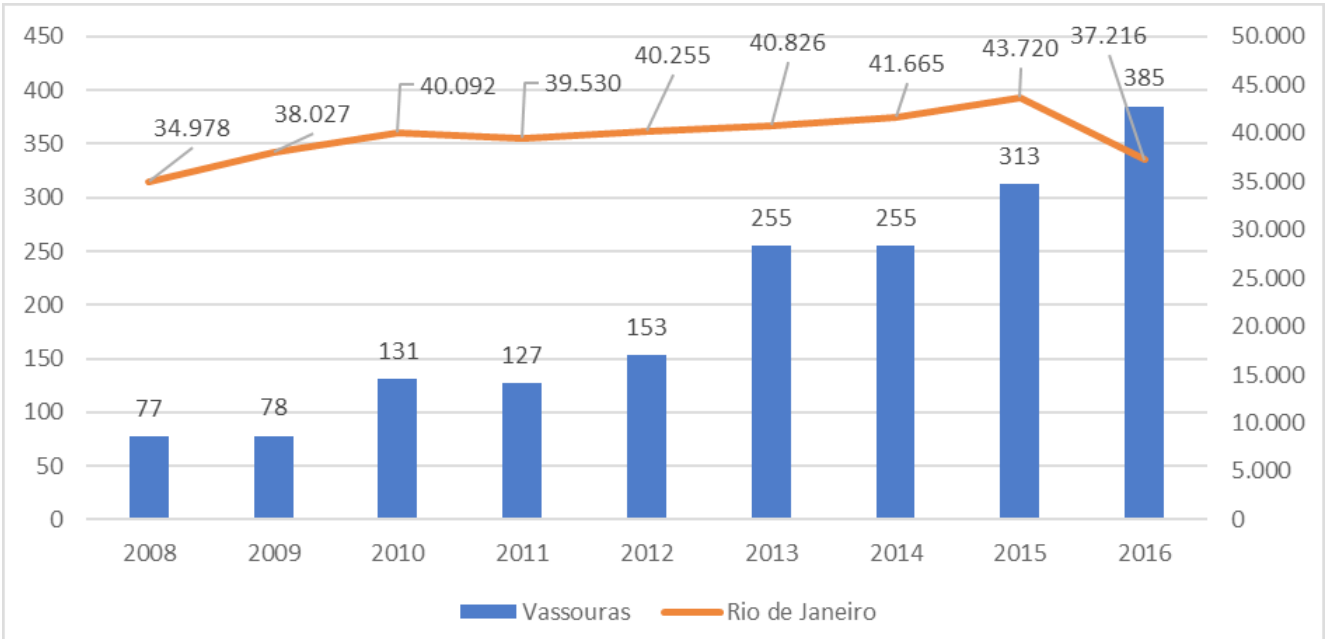

Figura 1. Número total de Novos Casos de Neoplasias em Vassouras e no Estado do Rio de Janeiro entre os anos de 2007 e 2016. No eixo vertical à esquerda, o número absoluto de casos de câncer na cidade de Vassouras. No eixo vertical à direita, o número absoluto de casos de câncer no estado do Rio de Janeiro. Na linha horizontal, encontra-se os anos estabelecidos para análise. 
Tabela 2: Valores de Indicadores 30a de Saúde em Vassouras e no estado do Rio de Janeiro, entre os anos de 2009 e 2016.

\begin{tabular}{|c|c|c|c|c|c|c|c|c|}
\hline & 2009 & 2010 & 2011 & 2012 & 2013 & 2014 & 2015 & 2016 \\
\hline Vassouras & $\uparrow 1 \%$ & $\uparrow 40,4 \%$ & $\downarrow 3,1 \%$ & $\uparrow 16,9 \%$ & $\uparrow 40 \%$ & $0 \%$ & $\uparrow 18,5 \%$ & $\uparrow 19 \%$ \\
\hline Rio de Janeiro & $\begin{array}{c}\uparrow \\
8,1 \%\end{array}$ & $\uparrow 5,4 \%$ & $\downarrow 1,4 \%$ & $\uparrow 1,8 \%$ & $\uparrow 1,4 \%$ & $2 \%$ & $\uparrow 4,9 \%$ & $\downarrow 14,8 \%$ \\
\hline
\end{tabular}

encontrados na cidade.

Os valores do Indicador $30 \mathrm{a}$, preconizado pelo SISPACTO, mostram o alcance da meta em apenas três momentos em Vassouras: 2009, com aumento de $1 \%$ em relação a 2008; 2011, com redução de $3,1 \%$; e 2014, quando não houve aumento ou diminuição em relação a 2013. Desde então, há aumento anual maior que nove vezes o estimado pela meta do indicador. No que se refere às metas municipais de pactuação, a meta é de 8 ao indicador, estando, ainda assim, em diversos anos, fora da meta.

Sobre os dados prévios de 2017, meses de janeiro a abril, podemos perceber um aumento sensível ( $\mathrm{p}<$ $0,005)$, quando comparado com o mesmo período nos anos de 2016 e 2015, como pode ser visto na Figura 2.

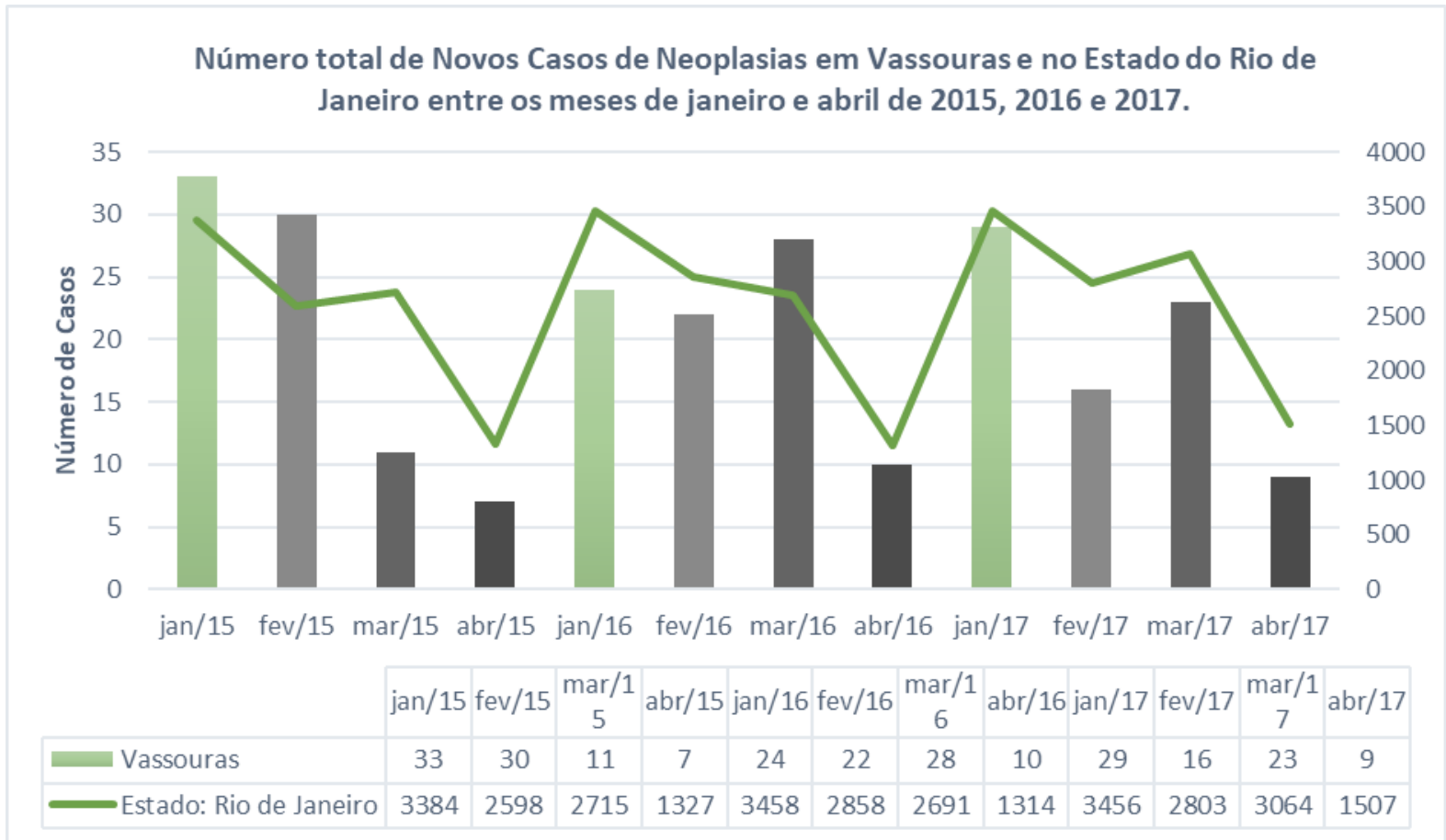

Figura 2. Número total de Novos Casos de Neoplasias em Vassouras e no Estado do Rio de Janeiro entre os meses de janeiro e abril de 2015, 2016 e 2017. No eixo lateral à esquerda, número absoluto de casos do município de Vassouras. No eixo lateral à direi-ta, número absoluto de casos do estado do Rio de Janeiro. No eixo horizontal, os meses em cada ano avaliados.

\section{Discussão}

O controle do número de casos de câncer vem se tornando um assunto recorrente nas mesas de debate internacionais. A evolução das metas e propostas de prevenção vem mudando constantemente, e, atualmente, vários fatores são analisados além da prevenção primária, como o bem-estar do paciente e prognóstico pós-tratamento ${ }^{3}$.
Um grande exemplo disso é a mudança do screening de câncer de próstata, quando se percebeu que, apesar do aumento do número de diagnóstico - influenciado, principalmente, pelo exame de PSA sérico - não houve repercussão na diminuição da mortalidade geral devido à doença $a^{3,5}$. Com isso, discutese muito até que ponto é necessário fazer prevenção primária do câncer de próstata e necessidade ou não de procedimentos pós-diagnóstico.

Esse tipo de abordagem ao câncer já é bastante 
comum no meio médico, seja no manejo da doença ou até mesmo nas síndromes paraneoplásicas: a detecção precoce do câncer não deve ser todo o enfoque do combate ao câncer ${ }^{1,8}$

Um dos maiores impulsos para o combate ao câncer foi a criação do CNCC - Campanha Nacional do Combate ao Câncer. Seguindo as ideias da Campanha Contra à Tuberculose, o surgimento da CNCC em dezembro de 1967 representou uma união nacional de diferentes órgãos para impulsionar a luta contra a doença. Segundo Decreto 61.9688, de 22/12/1967, a organização teria como objetivo "intensificar e coordenar, em todo o território nacional, as atividades públicas e privadas de prevenção, de diagnóstico precoce, de assistência médica, de formação de técnicos especializados, de pesquisa, de educação, ação social e de recuperação relacionadas com as neoplasias malignas em todas as suas formas clínicas, com a finalidade de reduzir-lhes a incidência"'.

Nota-se que há muito tempo há a preocupação em âmbito nacional com o combate ao câncer. Atualmente, o Ministério da Saúde institui a Política Nacional para a Prevenção e Controle do Câncer na Rede de Atenção à Saúde das Pessoas com Doenças Crônicas no âmbito do Sistema Único de Saúde (SUS), através da Portaria $\mathrm{N}^{\mathrm{o}} 874$, de 16 de maio de $2013^{9}$. Nesta, objetiva-se uma política de redução da mortalidade e da incapacidade causadas pela doença e a possibilitar a redução da incidência de alguns tipos de câncer, além de contribuir para a melhoria da qualidade de vida dos usuários com a enfermidade, por meio de ações de promoção, prevenção, detecção precoce, tratamento oportuno e cuidados paliativos ${ }^{7,8}$.

A portaria ${ }^{9}$ traz ainda os princípios e diretrizes relacionadas à comunicação em saúde para a formulação das estratégias que permitam a disseminação de conhecimentos sobre o câncer a profissionais e à população em geral.

Através do INCA, há ações e programas concisos em alguns tipos de neoplasias e fatores predisponentes. Dentre eles, pode-se citar Programa Nacional de Controle do Tabagismo; Controle do Câncer do Colo do Útero; e Controle do Câncer de Mama. Tais estratégias ajudam na promoção em saúde populacional, não somente através da elaboração de logística que permita uma excelente qualidade aos exames que visam o diagnóstico da doença, como também ajudam na divulgação à sociedade sobre os fatores de prevenção ${ }^{10}$.

Em Vassouras/RJ, a política de controle vem funcionado de modo interdisciplinar, respeitando a realidade da saúde nacional no aspecto condizente à importância do foco na saúde da mulher e do homem. Vários fatores atuam como vertentes positivas na cidade. Por ser um município com poucos habitantes, considerando as grandes metrópoles brasileiras, e haver uma universidade com cursos da área de saúde,
Vassouras tem uma rica possibilidade de métodos de ação e programas de prevenção e informação sobre as doenças crônicas não transmissíveis, como o câncer.

Através da Universidade de Vassouras, há a Liga Acadêmica de Oncologia, que produz e executa ações variadas de promoção de saúde sobre o câncer, desde feiras de saúde focando em Prevenção Primária, como também através do Projeto CUCA (Cura de Crianças com Câncer), projeto de extensão universitária focada em diagnóstico precoce e encaminhamento de neoplasias de crianças. Este tipo de estudo constituise como importante ajuda à população no suporte ao combate ao câncer.

Não obstante, a política em saúde do município também desempenha papéis importantes na luta contra o câncer, havendo, inclusive, liberdade para criação de novos métodos de controle entre os núcleos de Estratégia da Saúde da Família como, por exemplo, a adoção de cadernetas personalizadas que facilitam o acompanhamento por logradouro das mulheres que se enquadram na necessidade de acompanhamento de rastreio de câncer de colo de útero e que se encontram em situação de atraso do exame.

Todas essas propostas municipais, sejam através da própria estratégia da saúde do município, como também através de iniciativas de setores educacionais, atuam como fatores benéficos ao controle do câncer ${ }^{3,7,11}$.

Entretanto, há processos que podem acabar por não permitir a redução apropriada da incidência de neoplasias no município ao índice apropriado. $\mathrm{Na}$ Figura 1, percebe-se que em boa parte da linha de acompanhamento dos números de casos de neoplasias ao decorrer dos dez anos diverge da ascensão das colunas, possibilitando o entendimento de que o controle não é uniforme nas duas situações propostas. Isso se comprova na Tabela 2, quando se percebe que o valor do indicador 30a não apresenta constância entre as duas regiões.

Isso ocorre devido as estratégias de prevenção primária das neoplasias que não focam na epidemiologia atual do câncer no município. Não há preconização, além da adoção do exame de colonoscopia e sangue oculto nas fezes para detecção precoce de neoplasia de cólon, para as neoplasias visualizadas no sexo masculino, categoria historicamente mais difícil de se abordar nas estratégias de saúde ${ }^{11,12}$.

Enquanto na epidemiologia nacional, o câncer de próstata, pulmão e cólon se destacam, no município os cânceres mais prevalentes são os de cólon, câncer de estômago, câncer de próstata (em $5^{\circ}$ lugar) e câncer de bexiga (em $2^{\circ}$ lugar) ${ }^{6}$.

Vale ressaltar nessa epidemiologia o número de casos de câncer de bexiga, que levanta questões importantes acerca de doença ocupacional $1^{13,14,15}$.

De forma holística, percebe-se que há a evidente necessidade de entendimento, melhoria e acolhimento da saúde do homem à realidade de sua epidemiologia. 
Sobre a saúde da mulher, as neoplasias mais frequentes estão de acordo com a epidemiologia nacional sendo importante rever a adesão da população aos programas de saúde do município. Sabe-se, inclusive, que a taxa de adesão da população feminina ao exame Papanicolau vem diminuindo gradativamente, facilitando o aumento de neoplasias de colo de útero ${ }^{16}$. Por outro lado, vale ressaltar também a elevada taxa de neoplasias do trato digestivo, que ocupam o $1^{\circ} \mathrm{e}$ $2^{\circ}$ lugares de neoplasias mais frequentes em 2016 do sexo feminino em Vassouras. É preciso entender se o aumento dos casos é devido a uma política mais concisa de acompanhamento da população mais idosa na prevenção precoce do câncer de intestino ou isso se dá por uma maior incidência de fator causal.

Em um aspecto geral, a política de saúde no combate ao câncer deve ser revista, tomando como base seu aumento ao longo de uma década e na análise de aumento de casos, contrário ao observado no Estado do Rio de Janeiro, no primeiro trimestre de 2017. No que diz respeito às neoplasias com formas de intervenção já bem estabelecidas, como de próstata, mama, colorretal e colouterino, a melhor maneira de intervir frente a esses programas é trabalhando na sua divulgação e cumprimento. Isso pode se dar através de promoção em saúde direta à população para esses programas em específico, focando, em curto prazo, a diminuição da mortalidade dessas patologias e, a longo prazo, redução dos casos detectados por baixa incidência. Esse tipo de ideal é o mesmo encontrado no Canadá ${ }^{17}$, que visa em sua estratégia de controle a implementação de métodos usuais com eficácia, focando não só nas percepções de curto prazo, mas, sobretudo, em longo prazo, o qual eles referem ser de 25 anos. Às neoplasias que não se tem estabelecidos programas de controle específicos, o primeiro tipo de análise a ser feito é acerca da origem do aumento desse tipo de neoplasia, se há algo regional correlacionado com a doença. Em segundo, aumentar ainda mais os programas e ações que visam a diminuição holística de doenças crônicas não transmissíveis, como é o caso do programa de controle do tabagismo (sendo o tabagismo, inclusive, o maior fator de risco para câncer de bexiga).

\section{Considerações finais}

A epidemiologia mundial das principais causas de morbidade e mortalidade permite atribuir ao câncer uma posição de importância nas políticas de saúde pública. Em Vassouras, RJ, o aumento da sua incidência, divergente à velocidade de crescimento do número de casos estadual, levanta uma bandeira vermelha para a política e estratégias de saúde em combate ao câncer. Entender melhor como a epidemiologia da população se mostra facilita extremamente os focos de programas em saúde que visem a prevenção e combate da doença. Percebe-se, através dessa análise, que a evolução do câncer em Vassouras se deu de forma ímpar, necessitando de adaptações para sua epidemiologia local para que haja um melhor controle nos fatores causais e, assim, uma melhor adesão da população às políticas de saúde do município a curto prazo e uma melhora, em longo prazo, da incidência da doença.

\section{Agradecimentos}

À FUSVE/USS pela bolsa PIBIC/FUSVE, e à Coordenação de Pesquisa da Universidade de Vassouras.

\section{Referências}

1. Guerra MR, Gallo CVM, Mendonça GAS. The risk of cancer in Brazil: tendencies and recent epidemiologic studies. Revista Brasileira de Cancerologia 2005;51(3): 227-34.

2. Azevedo SG, Justina GC, Reis GV, Gonçalves VJ. Cancer mortality trends in Brazilian state capitals and other municipalities between 1980 and 2006. Rev Saúde Pública 2011;45(6): 1009-18.

3. Parada R, Assis M, Silva RCF, Abreu MF, Silva MAF, Dias MBK, et al. Brazilian cancer control policy and the role of primary care in cancer prevention and control. Rev. APS abr./jun. 2008;11(2): 199-206.

4. Ministério Da Saúde. Instituto Nacional de Câncer José Alencar Gomes Da Silva (Inca). Estimativa 2016: Incidência de Câncer no Brasil. Rio de Janeiro: Inca, 2015. Disponível em: http://www.inca.gov.br/estimativa/2016/ estimativa-2016-v11.pdf. [Acesso em: 05 jun., 2017].

5. Castro HAS, Iared W, Shigueoka DC, Mourão JE, Ajzen S. Contribution of PSA density in the prediction of prostate cancer in patients with PSA values between 2.6 and 10.0 ng/ml. Radiol Bras. Jul/Ago 2011;44(4):205-9.

6. Departamento de Informática do SUS (DATASUS). Banco de Preços em Saúde v2.3.0. Disponível em: http://aplicacao.saude.gov.br/bps/login.jsf. [Acesso em: 06 jun., 2017]

7. Teixeira LA, Porto M, Habib PABB. Public policies for cancer control in Brazil: elements of a trajectory. Cad. Saúde Colet 2012;20(3): 375-80. 8 Teixeira LA. O controle do câncer no Brasil na primeira metade do século XX. História, Ciências, Saúde - Manguinhos jul 2010;17(1): 13-31.

9. Ministério da Saúde (Brasil). Portaria nº 874, de 16 de maio de 2013. Institui a Política Nacional para a Prevenção e Controle do Câncer na Rede de Atenção à Saúde das Pessoas com Doenças Crônicas no âmbito do Sistema Único de Saúde (SUS). Diário Oficial da União 16 mai 2013;Seção 1.

10. Ministério da Saúde. Instituto Nacional de Câncer (INCA). INCA - Ações e Programas; 2016. Disponível em: http://www2.inca.gov.br/wps/ $\mathrm{wcm} /$ connect/acoes programas/site/home/nobrasil [Acesso em 11 jun 2017]

11. Siqueira FA, Silva SO, Benevides PM, Almeida MSB, Lima TNB, Bisneto, FP. Promotion and Prevention in Male Sexual Health: Challenges of Jose Pinheiro 1 Family Health Teams. R bras ci Saúde 2011;15(2):191-200. 12 Gomes R, Nascimento EF, Araújo FC. Why do men use health services less than women? Explanations by men with low versus higher education. Cad. Saúde Pública, mar. 2007; 23(3):565-574.

13. Mattos IE, Koifman S. Cancer mortality among electricity utility workers in a Southeastern region Brazil. Rev. Saúde Pública 1996;30(6): 564-75. 14 Burguer M. Epidemiology and Risk Factors of Urothelial Bladder Cancer. Eur Urol 2012 EURURO-4659; 8.

15. Witjes JA. EAU Guidelines on Muscle-invasive and Metastatic Bladder Cancer: Summary of the 2013 Guidelines. European Urology 2014; 65(1): 778-92. 
16. Machado HS, de Souza MC, Gonçalves SJC. Câncer de colo de útero: análise Epidemiológica e Citopatológica no município de Vassouras-RJ. Revista Pró-UniverSUS. 2017 Jan./Jun;08(1): 55-61

17 Fairclough L, Hill J, Bryant H, Kitchen-Clarke L. Accelerating knowledge to action: the pan-Canadian cancer control strategy. Curr Oncol 2012 Apr;19(2): 70-7. 